\title{
Editoriale \\ Gli economisti aziendali di fronte alla riforma Gelmini: prime considerazioni sull'accesso ai concorsi
}

Alberto Quagli*

A breve il Ministero dell'Università e della Ricerca Scientifica (MIUR) emanerà un decreto contenente il dettaglio dei requisiti minimi previsti per l'accesso alle fasce di docenza, così come prospettato dall'art. 16, comma 3 lettere a) e h) della Legge 30 dicembre 2010, n. 240 (Legge Gelmini).

Tale Decreto verrà emanato sulla base dei pareri richiesti a tre organismi ministeriali: il Consiglio Nazionale per l'Università (CUN), l'Agenzia Nazionale di Valutazione del sistema Universitario e della Ricerca (ANVUR) e il Comitato degli Esperti per la Politica della Ricerca (CEPR). Si poteva presumere che i tre organismi, slegati tra loro, si esprimessero in modo difforme. In effetti i tre pareri presentano differenze molto marcate e siamo curiosi e un po' preoccupati circa le regoli finali che il Ministero intenderà stabilire. Riteniamo però opportuno comprendere le scelte operate dai tre organismi per valutare se, al di là delle specifiche differenze, emerge una tendenza comune, che potrebbe essere poi ripresa dal MIUR dandole forza di legge.

Di questi tre pareri nel prosieguo si sintetizzano soltanto le specifiche disposizioni riferite all'area 13 (Scienze Economiche e Statistiche) o, ancora più precisamente, ai settori disciplinari componenti l'Accademia Italia di Economia Aziendale: SECS P07, (Economia Aziendale), P08 (Economia e gestione delle Imprese), P09 (Finanza Aziendale), P10 (Organizzazione Aziendale), P11 (Intermediari Finanziari). Tra questi la nostra maggiore attenzione va al settore $\mathrm{P} 07$ poiché costituisce il naturale alveo di interesse per i lettori di questa rivista.

I tre pareri sono molto diversi: il CUN ha emanato il documento ${ }^{1}$ nel quale si stabilisce che un candidato possa partecipare ai concorsi per le diverse fasce accademiche (ricercatore, professore associato, professore ordinario) solo se possiede un certo numero di pubblicazioni scientifiche. Con riferimento all'a-

* Editor di Financial Reporting.

1. http://www.cun.it/media/113040/do_2011_05_24.pdf. 
rea 13 stabilisce che per concorrere all'abilitazione di professore associato servono almeno sei pubblicazioni con il vincolo che almeno due di esse siano pubblicate in riviste, o con case editrici, di grande rilievo scientifico a diffusione internazionale negli ultimi cinque anni; per l'accesso al ruolo di professore ordinario servono almeno dieci pubblicazioni di cui almeno quattro siano pubblicate in riviste, o con case editrici, di grande rilievo scientifico a diffusione internazionale negli ultimi sei anni.

L'ANVUR $^{2}$ adotta invece il criterio della mediana, nel senso che ritiene possano partecipare ai concorsi solo coloro che sono sopra alla mediana come numero di pubblicazioni rispetto alla globalità dei docenti universitari pari fascia a livello nazionale. L'ANVUR a seconda dei settori disciplinari stabilisce poi diverse modalità di calcolare il numero di pubblicazioni. Con riferimento ai suddetti settori che compongono l'Economia Aziendale, l'ANVUR distingue i settori P08 e P10 dagli altri. I primi sono assimilati ai settori delle scienze mediche e tecnologiche considerando valide solo le pubblicazioni censite in alcuni database internazionali quali ISI o Scopus. Per gli altri settori (P07, P09, P11) si prescinde invece dalla inclusione in tali database e si considerano le pubblicazioni presenti nel database nazionale (oggi nel data base CINECA del MIUR, in futuro nell'Anagrafe delle Pubblicazioni Scientifiche). Le diverse pubblicazioni sono computate attribuendo a ciascuna un peso diverso in funzione della natura del lavoro:

1. monografia pubblicata presso editore internazionale = peso 3;

2. articolo pubblicato su rivista internazionale (ISI o Scopus) $=$ peso 1,5 ;

3. curatela di libro pubblicato presso editore internazionale $=$ peso 1,2 ;

4. monografia pubblicata presso editore italiano = peso 1 ;

5. altri articoli (internazionali non ISI o Scopus, italiani) e capitoli su libri italiani) $=$ peso 0,5 .

Il CEPR nel suo documento "Criteri e parametri di valutazione della produttività scientifica", con riferimento all' area 13, stabilisce che per il concorso da professore associato serva, in termini di pubblicazioni, "mediamente almeno una monografia significativa, e inoltre, mediamente, almeno un lavoro per ogni anno dopo il dottorato (fanno media anche i lavori pubblicati prima del conseguimento del dottorato)", mentre per professore ordinario servano "mediamente, almeno due monografie molto impegnative o una monografia e almeno due saggi di rilevante ampiezza pubblicati nelle principali riviste del settore, in Italia o all'estero; inoltre, mediamente, almeno un lavoro per ogni anno (non è chiaro se degli ultimi 5)".

Sono molte le osservazioni che emergono dalla lettura dei tre documenti. Mi limito in questo editoriale solo a due questioni.

2. http://www.anvur.org/media/277/documento01_11.pdf. 
a) la logica della peer review;

b) l'orientamento alla internazionalizzazione.

a) Tra i pochi punti in comune dei tre documenti emerge l'importanza del meccanismo della peer review. Ciascuno dei tre pareri stabilisce chiaramente che i lavori sono scientifici nella misura in cui siano stati accettati alla pubblicazione dopo un vaglio compiuto dai referee. Il referaggio, come stabilisce il CUN, deve essere anonimo e indipendente.

Mentre tale meccanismo è ben oliato quando uno studioso propone un suo lavoro ad una prestigiosa rivista internazionale, chi scrive ha la sensazione che sia non solo opportuno ma necessario nell'ambito delle discipline italiane di Economia Aziendale precisare chiaramente da parte delle competenti associazioni (AIDEA in primis) le caratteristiche del referaggio.

A mio avviso è infatti necessario precisare che l'indipendenza del referaggio sussiste nella misura in cui la scelta dei revisori da parte del responsabile scientifico avvenga: 1) solo in base alla competenze possedute del revisore in relazione all'argomento del lavoro, 2) evitando ovviamente qualunque influenza ex ante o ex post del responsabile scientifico sul revisore, 3) evitando di indicare revisori che per ovvie circostanze possano agevolmente individuare l'autore dell'opera sottoposta al loro esame ed essere conseguentemente condizionati (in positivo o in negativo) nel loro giudizio e 4) attribuendo al giudizio dei revisori un peso decisivo per l'accettazione del lavoro, pena l'inutilità dell'intera procedura.

Inoltre è opportuno che si precisi che il referaggio debba essere anche costruttivo, carattere che spesso, anche nelle migliori riviste internazionali, non sempre è rispettato. Il revisore deve cioè riuscire a far migliorare il paper e il suo autore, anche laddove sia convinto della inadeguatezza per la pubblicazione. Il merito di una rivista sempre più è sulle spalle dei revisori. In una monografia, poi, il lavoro del referee è ancora più pesante e importante, tenuto conto della ampiezza della review. Proprio per incentivare i revisori a compiere un buon lavoro, è necessario anche che sia riconosciuta nel curriculum a livello accademico la rilevanza del ruolo di referee ricoperto, magari tramite segnalazione da parte dei responsabili scientifici delle riviste o delle collane. Per evitare comportamenti difformi tra le riviste accreditate dall'AIDEA è ormai divenuto indispensabile che i tratti suddetti del referaggio e le ricadute (sperabilmente positive) per i revisori siano comunicati a tutti gli editor interessati.

b) Circa l'orientamento internazionale, rileviamo la notevole diversità di approcci tra i tre pareri sopra discussi: molto forte quello del CUN, decisamente segmentato quello dell'ANVUR, più blando quello del CEPR. Chi scrive ha partecipato ad una ricerca, in attesa di pubblicazione su altra rivista $^{3}$ dalla quale emerge che nel triennio 2008-2010 tra i docenti P07 strut-

3. Francesco Avallone, Alberto Quagli, Paola Ramassa, "La produzione scientifica dei 
turati nei ruoli universitari che hanno pubblicato almeno un lavoro (anche a più nomi) in una rivista inclusa nel gruppo del Journal rating AIDEA, per il settore Accounting \& Control (di più diretto interesse per il raggruppamento disciplinare P07) sono stati 19 gli associati e 21 i ricercatori, segno di una presenza di pubblicazioni internazionali ancora molto limitata.

Da tali dati è facile ricavare che l'asticella posta del CUN (due lavori internazionali per il concorso da associato come requisito minimo e quattro per gli ordinari) sembri allo stato attuale "eccessivamente elevata", al punto da ritenere che per i concorsi di prima fascia possano non esservi candidati, se il Ministero si adeguasse a quanto richiesto dal CUN. Riteniamo peraltro che neppure le indicazioni del CEPR possano essere accettate, dal momento che non pongono come criterio minimo neppure una pubblicazione internazionale. Questi sono i due eccessi da evitare. Più equilibrata la posizione dell'ANVUR anche se è criticabile per il fatto che i pesi alle diverse tipologie di lavoro sono stati attribuiti senza discriminare situazioni ben diverse: entro lo stesso ISI vi sono riviste internazionali su cui è molto difficile pubblicare che potrebbero avere tranquillamente peso pari a $5 \mathrm{o}$ 6 , mentre certe monografie italiane sembrano più degli instantlbook concorsuali che lavori scientifici veri e propri e un peso di 1 attribuito ad esse in modo egualitario costituisce un vero e proprio regalo.

Inevitabilmente sembra opportuno che il numero e il livello di pubblicazioni internazionali richieste per l'ammissione ai concorsi per i ruoli universitari nell'ambito economico-aziendale debba essere previsto fin da subito ma con un livello di difficoltà accettabile ad oggi (come AIDEA è stato proposto un articolo per i professori associati e due articoli per i professori ordinari) e crescente nel tempo, onde assecondare un processo di conversione delle politiche di pubblicazione dai lavori destinati alla sola comunità scientifica nazionale a lavori aperti al confronto internazionale.

L'argomento è molto complesso e merita molto più spazio. Certo è che le decisioni del MIUR nel prossimo Decreto saranno molto importanti e meriteranno un adeguato commento appena saranno rese note.

Passando ad argomenti più piacevoli, il 16 e il 17 Giugno si è svolto a Firenze, presso la Facoltà di Economia, il II workshop annuale della nostra rivista. Anche in questo caso i "numeri" sono stati buoni: 59 gli abstract ricevuti di cui ne sono stati selezionati 41. Dagli abstract sono discesi quindi 32 paper tra i quali sono stati infine scelti i 20 da presentare nelle sessioni parallele, dieci in italiano e dieci in inglese. Tra i paper presentati, una giuria ha assegnato i due premi da 1.000 euro, offerti da Deloitte e dalla Facoltà di Economia dell’Università di Firenze: il premio per il miglior lavoro in lingua

docenti italiani di Economia Aziendale. Un'analisi esplorativa nel triennio 2008-2010”, presentata al II workshop di Financial Reporting, Firenze, 16-17 giugno 2011. 
è andato al paper "Market Incentives and Regulators' Activity Shaping Financial Information: An Analysis of the Net Debt Disclosure in Italy" di Nicco Moscariello, Michele Pizzo e Rosa Vinciguerra ed il premio per il miglior lavoro in italiano è andato al paper "Professione amministratori: interlocking directorship e qualità degli utili nelle imprese italiane quotate" di Claudia Arena. Dai 32 paper ricevuti sono derivate infine 23 articoli proposti per la rivista e sono certo che nei prossimi numeri avremo modo di leggere alcuni di quei lavori.

Mi sento di esprimere un ringraziamento ai membri del comitato scientifico di Financial Reporting, che si sono impegnati a fondo nei ruoli di reviewer o chairman oltre a Francesco Giunta, Preside della Facoltà di economia dell'Università di Firenze e anima dell'iniziativa. Un vero plauso spetta al gruppo di giovani che ha organizzato con rara efficacia il workshop fiorentino: Luca Bambagiotti Alberti, Laura Bini, Francesco Dainelli (responsabile dell'organizzazione), Elena Gori, Giacomo Manetti, Lorenzo Neri.

L'appuntamento per il III workshop annuale è fissato per giugno 2012 a Napoli, presso l'Università Parthenope, coordinatore locale Raffaele Fiume. Il prossimo meeting della rivista sarà però a Venezia, con il convegno a larga partecipazione internazionale "Accounting Renaissance" nei giorni 3-5 novembre 2011, per il quale sono già giunti 120 paper. 